\title{
640-slice DVCT multi-dimensionally and dynamically presents changes in bladder volume and urine flow rate
}

\author{
YUNSHAN SU ${ }^{1}$, KEWEI FANG ${ }^{2}$, CHONGWEN MAO $^{1}$, SHUTIAN XIANG $^{1}$, JIN WANG $^{1}$ and YINGWEN LI ${ }^{1}$ \\ ${ }^{1}$ Department of Radiology, The Second People's Hospital of Yunnan Province, Kunming, Yunnan 650021; ${ }^{2}$ Department \\ of Urology, The Second Hospital of Kunming Medical University, Kunming, Yunnan 650101, P.R. China
}

Received December 7, 2016; Accepted September 6, 2017

DOI: $10.3892 /$ etm.2017.5671

\begin{abstract}
The present study aimed to explore the application of 640-slice dynamic volume computed tomography (DVCT) to excretory cystography and urethrography. A total of 70 healthy subjects were included in the study. Excretory cystography and urethrography using 640-slice DVCT was conducted to continuously record the motions of the bladder and the proximal female and male urethra. The patients' voiding process was divided into early, early to middle, middle, middle to late, and late voiding phases. The subjects were analyzed using DVCT and conventional CT. The cross-sectional areas of various sections of the male and female urethra were evaluated, and the average urine flow rate was calculated. The 640-slice DVCT technique was used to dynamically observe the urine flow rate and changes in bladder volume at all voiding phases. The urine volume detected by 640-slice DVCT exhibited no significant difference compared with the actual volume, and no significant difference compared with that determined using conventional CT. Furthermore, no significant difference in the volume of the bladder at each phase of the voiding process was detected between 640-slice DVCT and conventional CT. The results indicate that 640 -slice DVCT can accurately evaluate the status of the male posterior urethra and female urethra. In conclusion, 640-slice DVCT is able to multi-dimensionally and dynamically present changes in bladder volume and urine flow rate, and could obtain similar results to conventional CT in detecting urine volume, as well as the status of the male posterior urethra and female urethra.
\end{abstract}

Correspondence to: Dr Yunshan Su, Department of Radiology, The Second People's Hospital of Yunnan Province, 176 Qingnian Road, Kunming, Yunnan 650021, P.R. China

E-mail:wwangred@sina.cn

Dr Kewei Fang, Department of Urology, The Second Hospital of Kunming Medical University, 374 Dianmian Dadao Road, Kunming, Yunnan 650101, P.R. China

E-mail: 2482099228@qq.com

Key words: computed tomography, dynamic volume, excretory cystography and urethrography, lower urinary tract

\section{Introduction}

The lower urinary tract consists of the bladder and the urethra. Generally, the male urethra is $16-22 \mathrm{~cm}$ in length, with an average inner diameter of 5-7 $\mathrm{mm}$, while the female urethra is $3-5 \mathrm{~cm}$ long, with an inner diameter of $\sim 6 \mathrm{~mm}$ (1). Conventional radiography, such as retrograde and excretory urethrography, has been used for a century as the standard imaging technique in conventional research on the morphology and function of the lower urinary tract $(2,3)$. Under certain circumstances, other imaging techniques, including ultrasound and magnetic resonance imaging (MRI) may be conducive to the diagnosis of the lower urinary tract diseases $(4,5)$. However, methods including excretory urography, retrograde urography and contrast urography have a number of defects, including the use of an invasive procedure or a lack of quantitative indicators $(6,7)$. Therefore, quantitative evaluation of the anatomical form, such as the dynamic contraction of the bladder and urethra during urination, may not be conducted thoroughly.

Computed tomography (CT) using a multi-row detector (for example, 64-row multi-slice spiral CT) enables fast image capture and the generation of 2-dimensional (2D) and 3D reconstructed images via computer post-processing, and this relatively novel diagnostic technique has been used to evaluate lesions of the lower urinary tract (8-10). As the most recent CT technique, 640-slice dynamic volume CT (DVCT) has excellent spatial and temporal resolution, a wide capture range and a fast data acquisition system, with volume capture that provides 3D and 4D reconstructions of the region of interest.

The aim of the present study was to investigate the application of 640-slice DVCT to the lower urinary tract during urination, and provide a novel means for the quantitative examination of organic or functional lesions of the lower urinary tract.

\section{Patients and methods}

Patients. In this study, 70 healthy subjects admitted to The Second People's Hospital of Yunnan Province (Kunming, China) from September 2012 to June 2016 were included, with 43 males, aged $50.91 \pm 12.87$ years, and 27 females, aged $49.76 \pm 11.08$ years. Certain exclusion criteria were applied: Given the potential harm caused by radiation, healthy subjects (men and women) who had future fertility plans were 
excluded, and so the subjects in this study were predominantly middle aged and elderly. In addition, patients with a history of diseases of the bladder and the genitourinary system were also excluded.

Prior to the examination, all subjects were informed of the entire scanning approach, the significance of the study, and the risks associated with the exposure to X-rays, confirmed no iodine allergies, and provided written informed consent. The study was approved by the hospital ethics committee of The Second People's Hospital of Yunnan Province.

Examination methods, scanning conditions, and image generation using 640-slice DVCT

Preparation prior to examination. All subjects were asked to urinate and to self-test the duration of urination. A high-pressure injector was used to rapidly inject $50 \mathrm{ml}$ iopamidol (Iopamiro; Bracco Imaging S.p.A., Milan, Italy; $370 \mathrm{mgI} / \mathrm{ml}$; $18.5 \mathrm{~g}$ iodine) via a cubital vein catheter. Subjects were asked to not to urinate until their bladder was sufficiently full (when they felt a strong urge to urinate), and to lie on the examination bed. A urinal bottle was attached to the distal end of the penis of each male subject, while a urinal bowl was placed under the buttocks of each female subject.

Examination method and scanning conditions. In the supine, lateral or semi-sitting position, with feet forward, double orientation (coronal and sagittal) scanning was performed as the patient breathed calmly, using a CT scanner (Aquilion One TSX-301A; Toshiba Corporation, Tokyo Japan). The upper bound of the single field of view included the upper edge of the bladder, limited by the detector's maximum width of $16 \mathrm{~cm}$, and the lower bound included the male posterior urethra and a part of the anterior urethra, as well as the full length of the female urethra. The Dy-Volume capture mode was employed, with a capture thickness of $0.5 \mathrm{~mm}$ and an interval of 5-10 sec. For patients with a self-tested duration of urination $\leq 60 \mathrm{sec}$, an interval of $5 \mathrm{sec}$ was used for data capture, while for that $>60 \mathrm{sec}$, an interval of $10 \mathrm{sec}$ was used. All the subjects continued to urinate. The scanning was stopped at the end of urination. The detailed detection method and settings used for 640-slice DVCT were as described in the manufacturer's instructions. The scanning process of excretory cystography and urethrography using 640-slice DVCT is shown in Fig. 1.

Image generation and post-processing. The volume data of all phases were imported into a Vitrea post-processing workstation (VPMC-13204 B; Vital Images, Inc., Minnetonka, MN, USA). The 3D images of the lower urinary tract were marked by threshold selection, to produce the multiplanar reconstruction (MPR) and 3D volume images. MPR is able to provide the sections from any angle, and $3 \mathrm{D}$ volume images are able to display the target structure in 3D from various directions. The continuous playback of whole-phase MPR enabled dynamic 2D analysis of the bladder excretion to be performed, and 4D motion images of the lower urinary tract were obtained by the continuous playback of 3D images.

Volume CT dose index (CTDIvol) and dose length product $(D L P)$. According to the initial diagnostic reference level for adult CT perfusion (CTP) scanning specified in this study (the reference value of effective absorbed dose was set as $19.8 \mathrm{mSV}$ used in abdominal CTP), the recommended dose for routine pelvic CT examination is a CTDIvol of $35 \mathrm{mGy}$ and DLP of $780 \mathrm{mGy} . \mathrm{cm}$, with an effective dose of $11.7 \mathrm{mSv}$. In the present study, during the process of urination, an average of 10 sets of images were captured. The mean CTDIvol was $27.4 \mathrm{mGy}$ and the mean DLP was 439 mGy.cm, with an effective dose of $6.585 \mathrm{mSv}$. The effective dose when $\leq 15$ sets were captured was $9.8775 \mathrm{mSv}$, which was in line with International Commission on Radiological Protection requirements (11).

Examination using conventional CT. For comparison, SOMATOM Sensation 16 CT (Siemens AG, Munich, Germany) was used to scan the bladder with the same methods as Aquilion One, as described above. The matrix was set to $512 \times 512$, the voltage was $100 \mathrm{kV}$ and current was set to $100 \mathrm{~mA}$.

Urination phase division and urine volume detection. The beginning and end of the movement of each structure (the bladder, female urethra and the proximal male urethra) were recorded. For quantitative measurement, the urination process was equally divided into the early, early to middle, middle, middle to late, and late voiding phases chronologically, and the urine volume and urine flow rate of each phase were measured. Following urination, the urine was collected and the urine volumes were recorded using a measuring cylinder.

Observation of the status of the male posterior urethra and female urethra. The curved multiplanar reconstruction technique (12) was employed to mark the male posterior urethra and a part of the anterior urethra, as well as the female urethra. For the male urethra, the maximum diameter and the cross-sectional area of the pre-prostatic (internal urethral orifice), the prostatic (verumontanum), the membranous and the spongy urethra were measured, respectively (Fig. 2A). For the female urethra, the maximum diameter and cross-sectional area of the internal, intermediate and external urethral orifices were measured, respectively (Fig. 2B). Among the different scan phases, it was possible for certain phases to show the absence of filling with the urethral contrast agent. In such cases, two deputy chief physicians jointly confirmed and selected a phase with the most evident filling of urethral contrast agent for measurement. All measured data of the lower urinary tract were statistically compared.

Statistical analysis. The data obtained in this study were presented as bar chart or line charts. Student's t-tests were used for statistical analysis on SPSS 18.0 software (SPSS, Inc., Chicago, IL, USA). The average value of each set was the result from three independent experiments, shown as the mean \pm standard deviation. $\mathrm{P}<0.05$ was considered to indicate a statistically significant result.

\section{Results}

The application of 640-slice DVCT increases the accuracy of detection of the bladder urine volume. No significant difference was observed between the total bladder urine volume of the 43 normal males determined by DVCT $(344.28 \pm 182.29 \mathrm{ml})$ 


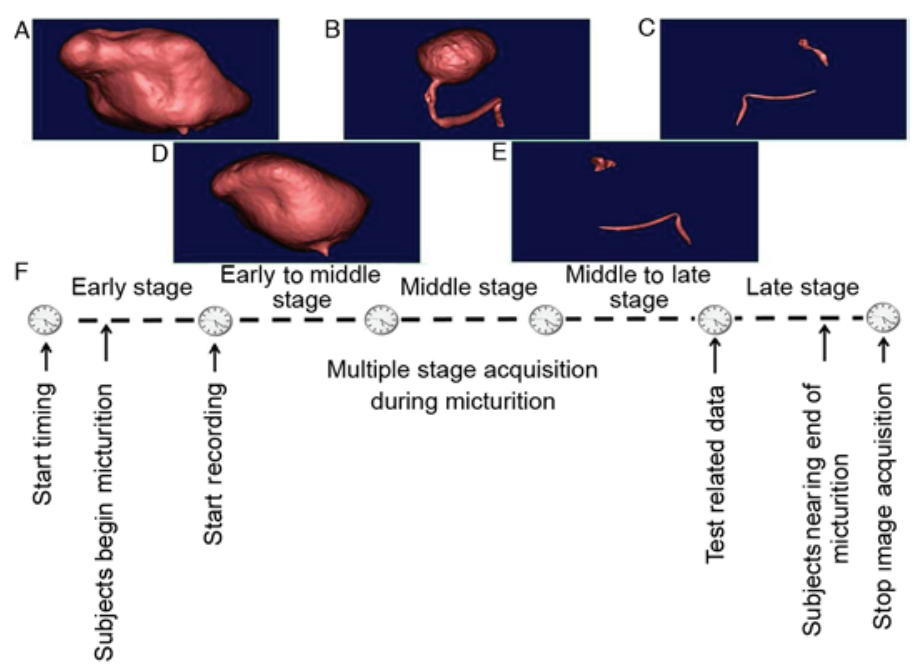

Figure 1. Flow chart for the imaging of the urinary excretion of the urinary bladder using DVCT. Images illustrate the urinary bladder at the (A) early stage, (B) early to middle stage, (C) middle stage, (D) middle to late stage and (E) late stage of urinary excretion, and (F) the imaging timeline. Every stage of the DVCT process was timed. DVCT, dynamic volume computed tomography.

A

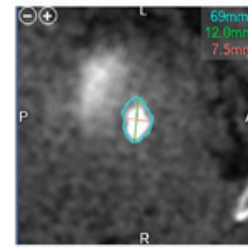

Pre-prostatic urethra
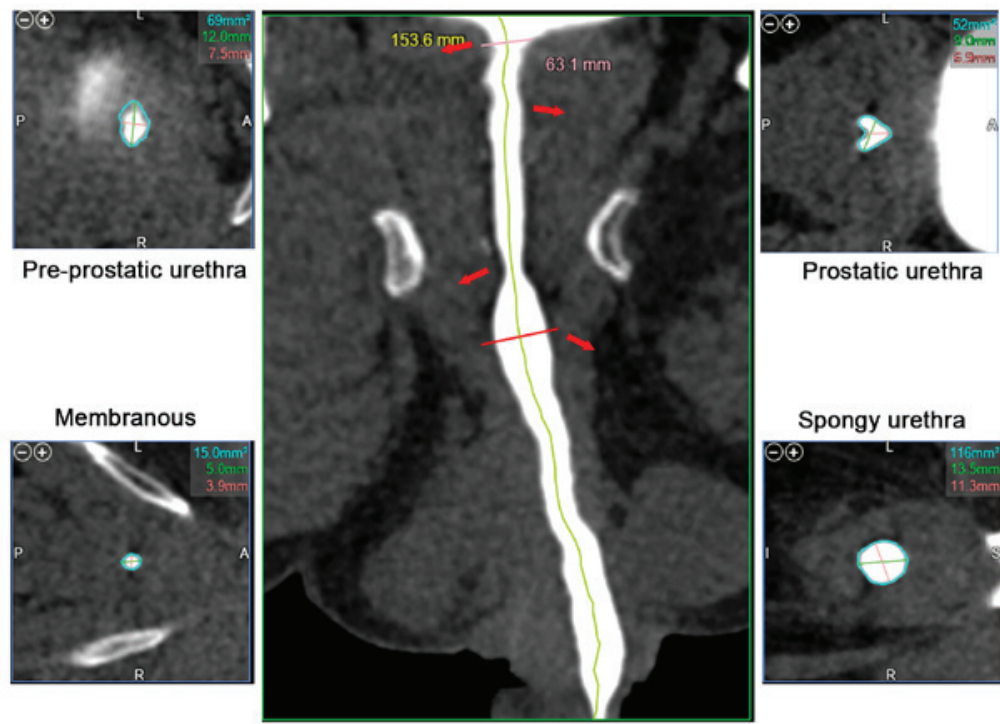

Prostatic urethra

Spongy urethra

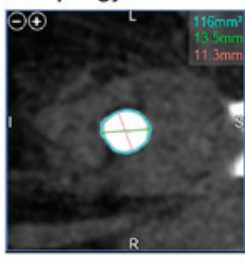

B

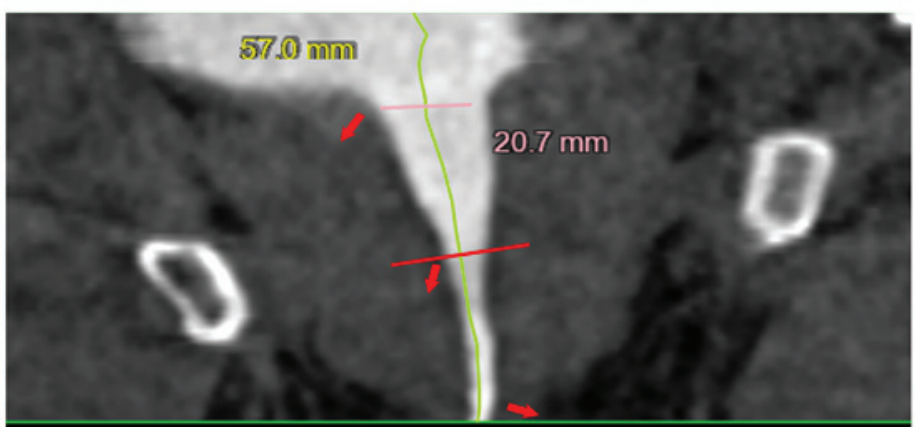

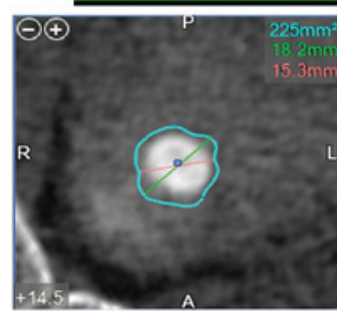

Internal urethra

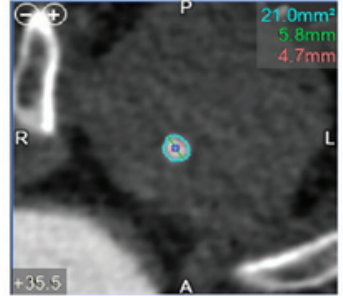

Intermediate urethra

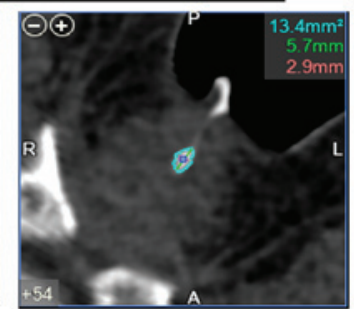

External urethra

Figure 2. Evaluation of the cross-sectional area of the male and female urethra. (A) Evaluation of the cross-sectional area of pre-prostatic, prostatic, membranous and spongy urethra for males. (B) Evaluation of the cross-sectional area of the internal, intermediate and external urethra for females. 
and the actual urine volumes $(351.79 \pm 178.36 \mathrm{ml})(\mathrm{P}>0.05)$. In addition, no statistically significant difference was identified between the total bladder urine volume of the 27 normal females determined by DVCT $(355.76 \pm 232.56 \mathrm{ml})$ and the actual urine volumes $(342.47 \pm 197.34 \mathrm{ml})$. However, the total bladder urine detected by conventional CT was not significantly different compared with the urine volume detected by 640-slice DVCT ( $>>0.05)$.

Using 640-slice DVCT dynamically observes the urine flow rate and the changes in the bladder volume at all voiding phases. Analysis of the data for the normal subjects demonstrated that males and females had their average maximum urine flow rate at the middle voiding phase. In particular, the average maximum urine flow rate at the middle voiding phase was $7.68 \pm 6.42 \mathrm{ml} / \mathrm{sec}$ for males, and $5.90 \pm 4.92 \mathrm{ml} / \mathrm{sec}$ for females. According to the statistical analysis, there was significant difference in the average flow rate at the late voiding phase between males and females. The calculated average urine flow rate at the late voiding phase was greater for females than for males, with the other phases exhibiting no significant difference (Fig. 3). Males and females had a peak flow rate in the middle voiding phase, with the flow rate presenting a bell-shaped distribution.

No significant difference in the bladder volume at all phases was detected between the 640-slice DVCT and conventional CT groups. Statistical analysis revealed that there was no significant difference in the bladder volume between normal males and females at all voiding phases (Fig. 4A). In addition, no significant difference was identified between the bladder volumes detected by DVCT and those detected by conventional CT (Fig. 4B).

640-slice DVCT evaluates the status of the male posterior urethra. The 640-slice DVCT and conventional CT methods were respectively employed to measure the maximum diameter and the cross-sectional area of the pre-prostatic, the prostatic, the membranous, and the spongy regions of the male urethra, and the results were statistically analyzed. The results exhibited no significant difference between the values measured by 640-slice DVCT and those measured using conventional CT (P>0.05; Fig. 5A).

640-slice DVCT evaluates the status of the female urethra. The 640-slice DVCT and conventional CT methods were respectively employed to measure the maximum diameter and the cross-sectional area of the internal, intermediate and external orifice regions of the female urethra. No significant difference was detected between the values measured by 640 -slice DVCT and those measured by conventional CT (P>0.05; Fig. 5B).

\section{Discussion}

For studies of the lower urinary tract, conventional urethrography has consistently been used as the gold standard for nearly a century. It is a simple, easy and low-cost approach, as it requires only conventional X-ray imaging and the retrograde injection of diluted contrast agent to examine the lower urinary

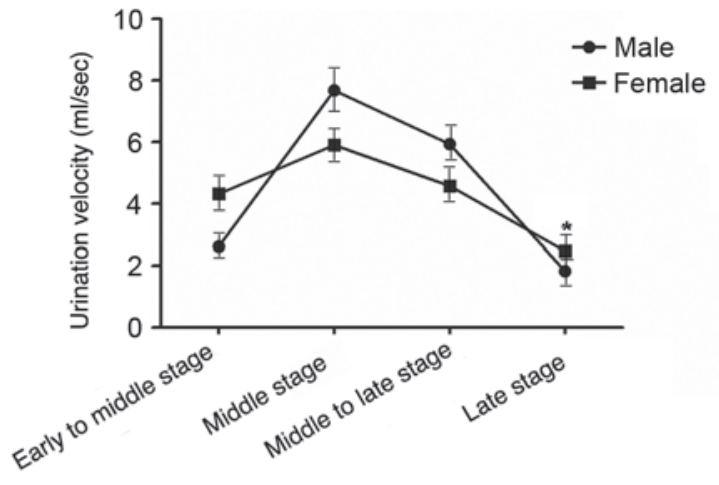

Figure 3. Urine flow rate and changes in the bladder volume at all voiding phases. ${ }^{*} \mathrm{P}<0.05$ for the male group vs. the female group.

tract $(13,14)$. However, since X-ray photographs obtained in the anteroposterior and double oblique views are overlapped images, and the procedure is invasive, the feasibility of this type of examination is limited $(4,15)$. Sonourethrography has been used to examine the lower urinary tract, but is not widely applied because of its small field of vision and a lack of recognition among urologists (4). MRI is also used to investigate pathological conditions of the lower urinary tract, but the inspection process is relatively complex $(5,15,16)$, and it is not suitable for use in subjects with a metal implant in the pelvic area.

CT urethrography (CTU) could obtain similar results to conventional urethrography (6-9). High-speed 64-slice spiral $\mathrm{CT}$ is able to scan the entire urethra and the bladder in $<6 \mathrm{sec}$. The introduction of contrast agents, as well as the high resolution and multi-dimensional post-processing capability of CT, even for subjects for whom a change in position is inconvenient, such as those with pelvic fractures, enables a full range of axial and multi-planar images of the lower urinary tract to be obtained in the supine position. Compared with conventional urethrography, CTU may be less painful for the subject being imaged. The comfortable position and high-quality 3D images substantially improve the compliance of CTU subjects. It is these advantages of CTU that enable male urethral injury and female urethral fistula to be examined using 64-slice spiral CT $(9,10)$.

In 640-slice MDCT, all volume data in a $16-\mathrm{cm}$ range are captured in $<0.35 \mathrm{sec}$ to generate time-point images. For example, when volumetric imaging of the bladder and urethra is conducted, dynamic volume images of the bladder and urethra are continuously captured by 640 -slice MDCT during urination, and measurements of the bladder and urethra are made at the early, early to middle, middle, middle to late, and late phases by division of the images according to time point, and collected for statistical analysis. Previous studies have reported the use of DVCT for imaging of the osteo-odonto-keratoprosthesis lamina, acute chest pain or calcified coronary atherosclerotic plaque and airway deformation (17-20). Therefore, in the present study, DVCT technology was applied to predict the bladder volume and urine flow rate of patients. The results revealed that there was no significant difference between the total bladder urine volumes of males and females measured using DVCT and the actual urine volumes. This demonstrated that 640 -slice 

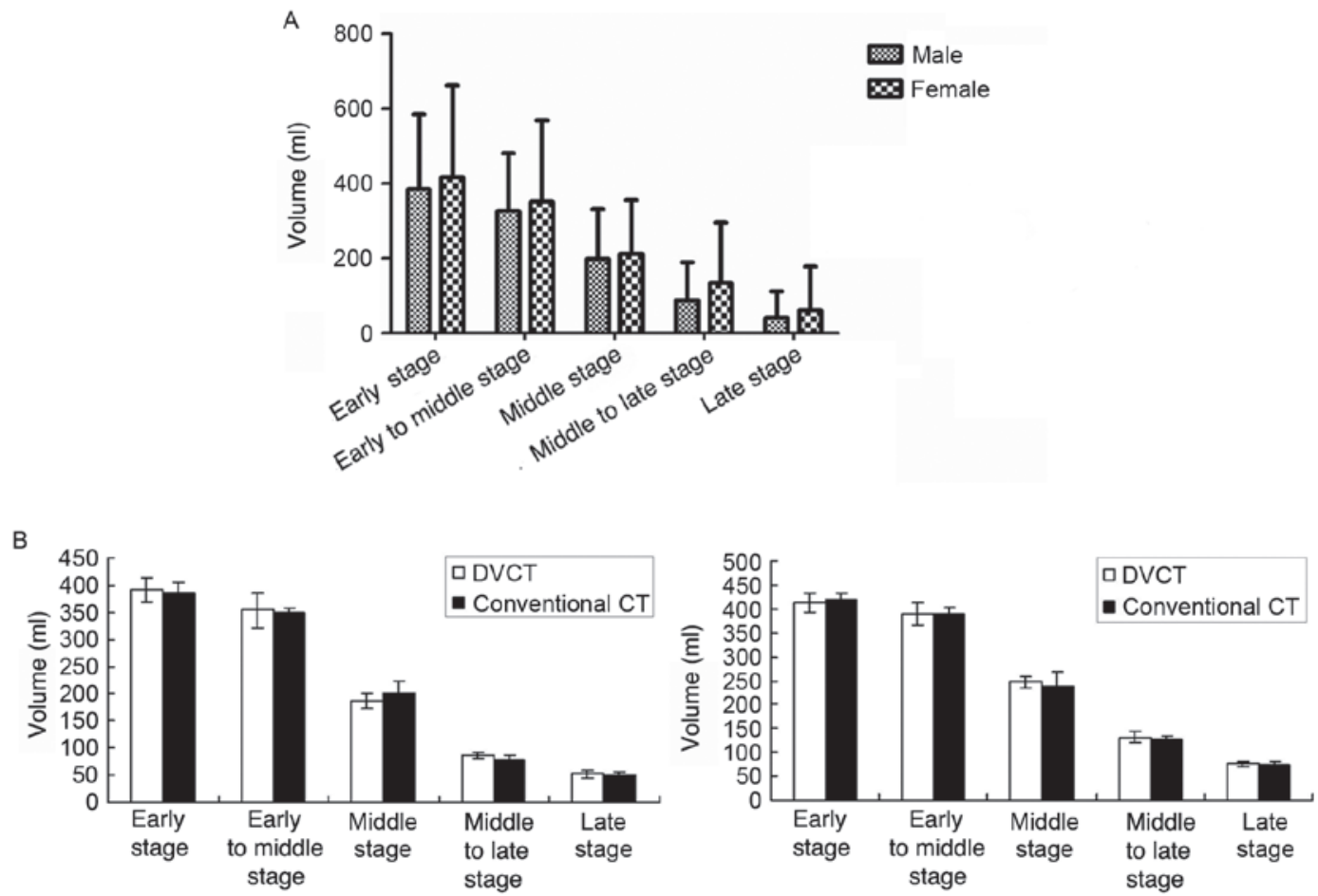

Figure 4. Bladder volume at all phases. (A) Bladder volume at all phases in males and females. (B) Bladder volume in male patients (left) and female patients (right) at all phases determined using 640-slice DVCT and conventional CT. CT, computed tomography; DVCT, dynamic volume CT.
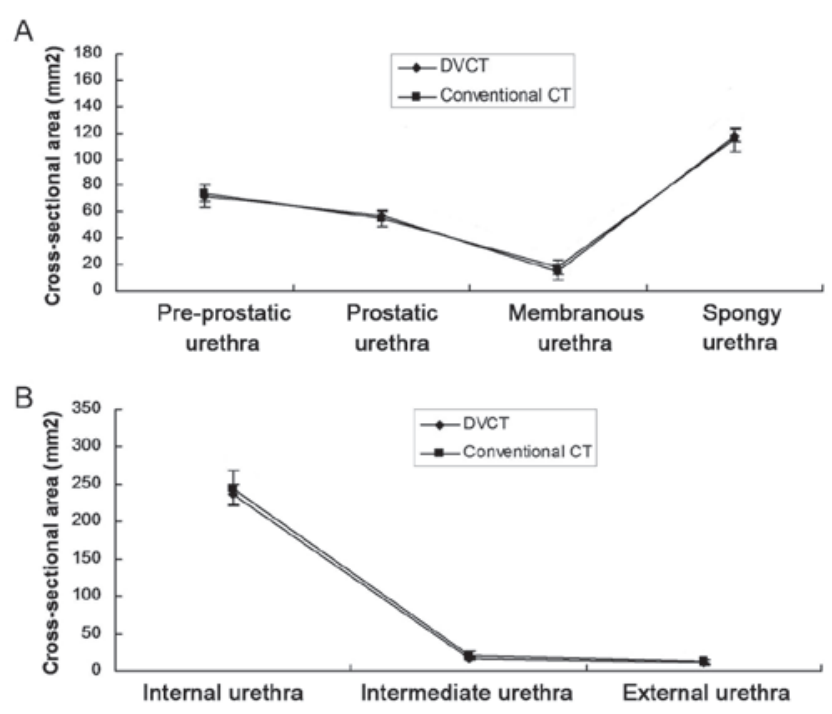

Figure 5. Cross-sectional area analysis of the male and female urethra using 640-slice DVCT and conventional CT. (A) Evaluation of the cross-sectional area of (A) male and (B) female urethral structures. CT, computed tomography; DVCT, dynamic volume CT.

MDCT was able to accurately capture isotropic data of the bladder and urethra and measure the volume values, displaying the anatomical data of the bladder and urethra authentically and quantitatively. In addition, a significant difference in the average flow rate at the late voiding phase between males and females was identified, with females having a higher calculated urine flow rate than males at the late voiding phase. The reason for this is possibly that female subjects have an active contraction of the detrusor at the late voiding phase. However, this requires further confirmation by further examinations.

The measurements in the present study focused on the cross-sectional area and the inner diameter of the urethral segments, which are previously unreported. To the best of our knowledge, measurements of the cross-sectional area and the inner diameter of each urethral segment during urination have not been reported previously. For this reason, the correlation between the widest diameter and the minimum diameter of the cross-sectional area and the functional evaluation of the urethra is expected to provide the basis for further research (21).

In conclusion, 640-slice DVCT is a promising examination method for the replacement of traditional imaging methods. Its advantage is that, by using only an approach and position acceptable to the subject, in conformity with the requirements of the radiation dose, data at each voiding phase is obtained, 3D and 4D images are reconstructed, the anatomy of the lower urinary tract is dynamically and accurately displayed, and intramural data of the lower urinary tract can be quantified. This method is likely to contribute to the diagnosis and quantitative assessment of diseases in the lower urinary tract with anatomical changes, in the absence of medical radiation exposure. This is the first examination of the lower urinary tract by the application of 4D CT and quantitative assessment, and a large number of future studies on various lower urinary tract diseases are required to determine the clinical value of excretory cystography and urethrography using 640-slice DVCT. However, due to the potential risks of exposure to radiation, although the radiation dose used for scanning was in the safe range, the use of the proposed method is not recommended for children and childbearing women, due to the special structure of their pelvic cavity. 


\section{Acknowledgements}

Financial assistance was provided by Yunnan Provincial Science and Technology Department (grant no. 2011FB229).

\section{References}

1. Zhu ZS, Wu H, Li RY and Wang DH: One-stage urethroplasty with circumferential vascular pedicle preputial island flap for perineal hypospadias. Zhonghua Zheng Xing Wai Ke Za Zhi 26: 258-261, 2010 (In Chinese).

2. Pavlica P, Menchi I and Barozzi L: New imaging of the anterior male urethra. Abdom Imaging 28: 180-186, 2003.

3. Kim B, Kawashima A and LeRoy AJ: Imaging of the male urethra. Semin Ultrasound CT MR 28: 258-273, 2007.

4. Gallentine ML and Morey AF: Imaging of the male urethra for stricture disease. Urol Clin North Am 29: 361-372, 2002.

5. Milosevic M, Voruganti S, Blend R, Alasti H, Warde P, McLean M, Catton P, Catton C and Gospodarowicz M: Magnetic resonance imaging (MRI) for localization of the prostatic apex: Comparison to computed tomography (CT) and urethrography. Radiother Oncol 47: 277-284, 1998.

6. Vilalta L, Altuzarra R, Espada Y, Dominguez E, Novellas R and Martorell J: Description and comparison of excretory urography performed during radiography and computed tomography for evaluation of the urinary system in healthy New Zealand white rabbits (Oryctolagus cuniculus). Am J Vet Res 78: 472-481, 2017.

7. Diefenderfer DL and Brightling P: Dysuria due to urachal abscessation in calves diagnosed by contrast urography. Can Vet J 24: 218-221, 1983.

8. Chou CP, Huang JS, Wu MT, Pan HB, Huang FD, Yu CC and Yang CF: CT voiding urethrography and virtual urethroscopy: Preliminary study with 16-MDCT. AJR Am J Roentgenol 184: 1882-1888, 2005.

9. Zhang XM, Hu WL, He HX, Lv J, Nie HB, Yao HQ, Yang H, Song B, Peng GM and Liu HL: Diagnosis of male posterior urethral stricture: Comparison of 64-MDCT urethrography vs. standard urethrography. Abdom Imaging 36: 771-775, 2011.

10. Qi Z, Lemen LC, Lamba M, Chen HH, Samaratunga R, Mahoney M and Hendrick RE: Radiation dose to the breast by 64-slice CT: Effects of scanner model and study protocol. Acad Radiol 23: 987-993, 2016
11. Cool DA, Lazo E, Tattersall P, Simeonov G and Niu S; International Commission on Radiological Protection: ICRP publication 125: Radiological protection in security screening. Ann ICRP 43: 5-40, 2014.

12. De Filippo M, Castagna A, Steinbach LS, Silva M, Concari G, Pedrazzi G, Pogliacomi F, Sverzellati N, Petriccioli D, Vitale M, et al: Reproducible noninvasive method for evaluation of glenoid bone loss by multiplanar reconstruction curved computed tomographic imaging using a cadaveric model. Arthroscopy 29: 471-477, 2013.

13. Theisen KM, Kadow BT and Rusilko PJ: Three-dimensional imaging of urethral stricture disease and urethral pathology for operative planning. Curr Urol Rep 17: 54, 2016.

14. Kawashima A, Sandler CM, Wasserman NF, LeRoy AJ, King BF Jr and Goldman SM: Imaging of urethral disease: A pictorial review. Radiographics 24 Suppl 1: S195-S216, 2004.

15. Osman Y, El-Ghar MA, Mansour O, Refaie H and El-Diasty T: Magnetic resonance urethrography in comparison to retrograde urethrography in diagnosis of male urethral strictures: Is it clinically relevant? Eur Urol 50: 587-593, 2006.

16. Oh MM, Jin MH, Sung DJ, Yoon DK, Kim JJ and Moon du G: Magnetic resonance urethrography to assess obliterative posterior urethral stricture: Comparison to conventional retrograde urethrography with voiding cystourethrography. J Urol 183: 603-607, 2010 .

17. Norris JM, Kishikova L, Avadhanam VS, Koumellis P, Francis IS and Liu CS: Comparison of 640-slice multidetector computed tomography versus 32-slice MDCT for imaging of the osteo-odonto-keratoprosthesis Lamina. Cornea 34: 888-894, 2015.

18. Yu SJ, Zhang L, Chen YF and Zhang J: Effects of heart rate on image quality and radiation dose of 'triple rule-out' 320-row-640-slice multidetector computed tomography scan in patients with acute chest pain. Zhonghua Yi Xue Za Zhi 92: 2652-2655, 2012 (In Chinese).

19. Kristanto W, van Ooijen PM, Groen JM, Vliegenthart R and Oudkerk M: Small calcified coronary atherosclerotic plaque simulation model: Minimal size and attenuation detectable by 64-MDCT and MicroCT. Int J Cardiovasc Imaging 28: 843-853, 2012.

20. Yin Y, Choi J,Hoffman EA, Tawhai MH and Lin CL: A multiscale MDCT image-based breathing lung model with time-varying regional ventilation. J Comput Phys 244: 168-192, 2013.

21. Watanabe H, Takahashi S and Ukimura O: Urethra actively opens from the very beginning of micturition: A new concept of urethral function. Int J Urol 21: 208-211, 2014. 